\title{
A traffic flow phase adaptive routing for vehicular communication on highways
}

\author{
Ping Wang ${ }^{1 *}$, Fuqiang Liu', Dawei Li ${ }^{1}$ and Nguyen Ngoc Van²
}

\begin{abstract}
Identification of traffic flow is very important since it can help provide dynamic navigation and optimize the performance of vehicular ad hoc networks (VANETs). The existing ways for estimating the traffic state mostly show the drawbacks of large computation and hard implementations. In this paper, we propose a self-organizing way to collect traffic information through VANET communications without any aid of infrastructures. Then, a method based on fuzzy logic is presented to predict the phase of traffic flow timely. This method shows the advantages of easier implementations and less computation, and it can adapt dynamically to take more traffic factors into consideration if needed. With the knowledge of current traffic status, a new routing algorithm for vehicular communication on highways is proposed, which can be adaptive to different phases of traffic flow and guarantee reliable transmission in different environments. The simulation results show that our self-organizing proposal gains satisfactory accuracy according to the real-time identification of traffic flow, and the network performance of the adaptive routing algorithm is improved compared with traditional routing algorithms in terms of packet delivery ratio and throughput; at the same time, the end-to-end delay is still within an acceptable level.
\end{abstract}

Keywords: VANETs; Routing; Traffic flow; Three-phase traffic model; Fuzzy logic

\section{Introduction}

Vehicular ad hoc networks (VANETs), as a novel fundamental platform for providing real-time information exchange for safety-related services, are expected to play an important role in future intelligent transportation systems (ITS). VANETs exhibit characteristics that are dramatically different from many generic MANETs due to mobility constraints, driver behavior, and high mobility. Through vehicle-to-vehicle or vehicle-to-infrastructure communication, they can provide ubiquitous connectivity while on the road to moving vehicles and thus improve road safety or traffic efficiency. For example, through VANET communications, they can help drivers avoid collisions and coordinate driving at the intersection, highway entrance, and other dangerous locations. This is why many government organizations and car manufacturers are carrying out extensive research in this field. We believe such efforts will revolutionize our traveling habits by increasing

\footnotetext{
* Correspondence: pwang@tongji.edu.cn

'Broadband Wireless Communication and Multimedia Laboratory, College of Electronics and Information Engineering, Tongji University, Shanghai 201804, China

Full list of author information is available at the end of the article
}

safety on the road while entertaining our feelings simultaneously [1].

Generally, there are two different kinds of transmission modes in a wireless network. One is point to point and the other is point to multipoint. The former is used to transmit data to just one receiver, and the latter is used to transmit data to multiple receivers simultaneously. Some applications need point-to-point transmission, such as gossip between two vehicles. Other applications need point-to-multipoint transmission such as pushing gas station information to all nearby vehicles. For both these two transmission modes, data should be transmitted via multihop communication. That means data will be forwarded by several relays before reaching the destination. The nature of most of these applications requires quick and reliable information delivery as well as robust end-to-end multihop throughput [2]. Because of the high mobility and unreliable channel condition, the communication links among vehicles on highways are easily disrupted. If the routing path is unstable, packets are easily discarded during the period of forwarding, which may cause repetitive transmission, and thus, transmission efficiency and system throughput are reduced. 
Different from other ad hoc networks, VANETs are highly mobile since traffic flow on highways changes rapidly with time and space. Maybe now a vehicle is moving freely with few neighbors. But in a few minutes, it may be congested with many vehicles around. Therefore, the topology of VANETs varies frequently, which makes a single routing strategy not adapt to the changing environments. Point-to-multipoint transmission may be suitable for freely moving vehicles, but not viable in traffic jam owing to possible broadcast storm. On the contrary, there is no problem for point-to-point transmission in traffic jam, but it does not perform well in free traffic flow since the transmission of one copy often fails. Therefore, during designing forwarding strategies for vehicular communication, the spatial-temporal states of traffic flow should be considered.

The prediction and identification of the states of traffic flow are important subjects in the field of intelligent transport systems [3,4], since they can not only help drivers improve traveling efficiency through dynamic navigation, but also help optimize the performance of VANETs. In previous studies, most of the data for traffic state prediction and identification were obtained through pre-deployed sensors as well as other infrastructures like ground coils. Such methods cost a lot, and the majority of the existing ways to estimate the traffic state show drawbacks of large computation and hard implementation. In this paper, we propose a new way for estimating traffic flow based on VANETs themselves. That is, traffic information is collected through VANET communications to reduce the costs effectively. Then, we present a prediction method based on fuzzy logic and membership functions which has advantages of easier implementation and less computation. Furthermore, to improve accuracy, this method can be adjusted dynamically to consider more traffic factors if necessary. To exclude the abnormal data which may cause the wrong results, data consistency test is conducted before state identification. The simulation results based on a traffic simulator and communication simulator show that the proposed self-organizing method for traffic phase prediction gains high accuracy.

Since the network behaves differently in different traffic phases, a single transmission mode may not perform stably under the changing VANET environment. Ducourthial et al. [5] suggest that better performances could still be obtained with some context-aware conditions, forged with a program that takes into account the traffic road characteristics. The experiment results in [6] also clearly indicate that designing a smart message dissemination strategy is fundamental in order to implement fast and reliable multihop message propagation. Based on the available traffic states, a routing algorithm adaptive to different traffic flows is proposed in this paper to improve forwarding performance. The point-to-point transmission is adopted in traffic jam to avoid broadcast storm in a stable networking environment. Owing to fragile connectivity in free traffic flow, the point-to-multipoint mode is used to guarantee a certain reliable transmission. Simulation shows that the proposed algorithm can improve packet delivery ratio and throughput under the scenarios with different traffic flows.

The remaining parts of the article are organized as follows. Section 2 describes the background of traffic state estimation and adaptive forwarding strategies in VANETs. Section 3 gives the self-organizing prediction of traffic flow based on the methods of fuzzy logic and data consistency test. Section 4 presents adaptive routing schemes based on different traffic flow phases. In Section 5, the performance study based on multiple simulation tools including a traffic simulator and communication simulator is presented. Finally, a conclusion is made in Section 6.

\section{Background}

More and more researchers are realizing that the system performance of VANETs can be improved by utilizing traffic conditions. To do this, first the current traffic conditions should be acquired in real time, which reflect the changes of network environments. Then, a corresponding forwarding strategy might be adopted. When changes in traffic conditions take place, the forwarding strategy would be adjusted accordingly.

\subsection{Traffic state estimation}

Currently, most of traffic state estimation approaches are designed for the infrastructure-based traffic information systems, which rely on detecting devices such as inductive loop detectors or traffic surveillance cameras to be installed at various locations. Consequently, they cannot be applied in rural areas or on highways without such infrastructures. With the development of wireless communication, a self-organizing vehicular traffic information system becomes an emerging technology, where the vehicles can collect and process the traffic information without relying on any fixed infrastructure. A distributed vehicle density estimation scheme [7] is proposed, where a road segment is divided into multiple fixed-size cells. The cell density is calculated by counting the number of vehicles in the cell via a designated vehicle. Through exchanging the information of cell density among the designated vehicles, the average density on the road is also acquired. Similar to [7], Panichpapiboon and Pattaraatikom [8] propose a neighbor-based scheme to estimate the vehicle density based on the number of vehicles in the vicinity of the probe vehicle. However, it does not require a fixed cell size. Based on the communication between vehicles and road side unit (RSU), Xue et al. [9] propose a detection scheme for traffic congestion. In order to detect the queue length (which acts as a measure index to 
evaluate the traffic status of intersections) timely and accurately, a restricted greedy forwarding strategy is put forward to transmit the status information from the tail vehicle via multihop broadcast. For these self-organizing traffic information systems, vehicles can obtain the traffic information distributed, and mainly, the vehicle density is involved. However, other traffic metrics such as velocity may also influence the network topology greatly. Therefore, how to characterize the non-uniform distribution of cars and velocity, and unique connectivity dynamics is required.

In the research of dividing traffic state, the three-phase traffic model has been widely researched and further developed by scholars around the world. The three-phase traffic model [10] is a classic traffic flow theory put forward by B. S. Kerner between 1996 and 2002. It focuses mainly on the explanation of the physics of traffic breakdown and resulting congested traffic on highways. Kerner uses three-phase traffic theory to describe traffic: free flow, synchronized flow, and wide moving jam (here, we use congested flow to represent wide moving jam). In free flow phase, vehicles are moving freely and there is almost no interaction between them. In this phase, vehicles are few and topology changes very rapidly. In synchronized flow phase, traffic density is increased. Vehicles do not move freely any longer, and the interaction between them is increased; theories such as car following can be used to represent vehicles' moving behavior. In congested flow phase, vehicles move very slowly and traffic density is generally very large. In such phase, topology nearly does not change. Apparently, in different phases, topology dynamics is very different, which will influence routing performance. Thus, the forwarding strategies for vehicular communication should be designed based on the phase of traffic flow.

\subsection{Forwarding strategies adaptive to different environments}

Considering that the underlying network topology of VANETs is determined by roads and by the characteristics of vehicular traffic on the roads, the performance of many forwarding strategies is improved by utilizing these properties. Resta et al. [6] made an important observation with regard to the dependence of the relative benefits of increasing channel reliability on traffic condition. This behavior seems to suggest that the relative benefit of implementing reliable one-hop communication decreases with car density. Hence, network designers might design density-aware reliable one-hop communication primitives, where the desired value of reliability is tuned depending on the observed car density. In [11], a transmission range is set dynamically according to local traffic conditions, which maintains connectivity effectively while minimizing the adverse effects of a high transmission power. Tonguz et al. [12] identify three very different regimes that a vehicular broadcast protocol needs to work on: (i) dense traffic regime, (ii) sparse traffic regime, and (iii) regular traffic regime. They build upon their previously proposed routing solutions for each regime, and the results show that the broadcast message can be disseminated efficiently. All of these schemes do not focus on improving forwarding performance according to different states of traffic flow, which will be addressed in this paper.

\section{Identification of traffic flow phase}

The dynamics of traffic flow causes a great impact on data transmission in vehicular ad hoc networks. In free flow, the vehicles are rare and move very quickly; thus, they may disconnect from each other frequently. In congested flow, every vehicle will have well-connected neighborhood. Therefore, a single method of data transmission cannot meet the demands of message forwarding under different traffic flow scenarios. For example, point-to-multipoint transmission which may be suitable in free flow will cause serious broadcast storm in congested flow. Thus, if the phase of traffic flow can be identified timely, an adaptive routing protocol based on traffic flow can be proposed to guarantee optimal performance under the frequently changing traffic environments on highways.

\subsection{Gathering local traffic information}

In previous researches, traffic information is usually gathered through pre-deployed sensors as well as other infrastructures, and this information should be transmitted to the vehicles timely, in which way large costs are produced. Especially in remote rural areas owing to the lack of these infrastructures, it becomes infeasible. With the development of wireless communication technologies, the module of wireless access becomes a standard configuration for a new vehicle. Thus, we can collect traffic information through VANET communications to reduce the costs effectively.

Each node needs to broadcast a HELLO message which contains its own information to all the directly connected neighbors. The information includes vehicle ID, velocity, moving direction, and its current position. Such kind of information can be obtained through on-board GPS. Upon reception of such HELLO message, a vehicle can collect the number of vehicles around and their speeds with the same moving direction. Then, it computes the average speed and local density. These kinds of traffic information will be used to identify the phase of traffic flow.

\subsection{Identification of traffic flow phase based on fuzzy logic}

Based on the local traffic information gathered through VANET communications, a fuzzy logic theory [13] is 
used to identify and predict the traffic flow phase. This method divides vehicular speed and density into three categories as defined in Kerner's three-phase traffic model. A membership degree is computed according to the membership function of fuzzy sets with testing data, which is called training process. Figures 1 and 2 give the membership functions of fuzzy sets based on vehicular speed and density, respectively.

For variable $x$, the predicted value of the $i$ th phase is calculated as follows $(i=1,2$, and 3 , which correspond to the phases of free flow, synchronized flow, and congested flow, respectively):

$$
\mu_{A i}(x)=\alpha \cdot \mu_{A i, y}(x)+(1-\alpha) \cdot \mu_{A i, \rho}(x)
$$

where $\mu_{A i, v}(x)$ and $\mu_{A i, \rho}(x)$ are the membership degrees of the $i$ th phase in terms of vehicular speed and density, respectively. $\alpha$ is the weighting factor. The larger the value of $\alpha$ is, the greater the role of speed will be. Otherwise, the smaller the value of $\alpha$ is, the greater the role of density will be. Considering the practice of the threephase traffic model, the speed has greater impact on traffic flow phase than the density under the same condition. Therefore, the value of $\alpha$ should be greater than 0.5 .

Among the membership degrees under the three traffic flow phases, that whose value is the biggest will represent the final predicted phase.

\subsection{Data consistency check}

The results of phase identification may be abnormal via designated vehicles. To improve accuracy, we calculate the mean speed as input and consider the impact of the speed of all vehicles among the wireless communication range. Besides, the distribution diagram method is used to remove the abnormal data.

For the distribution diagram method, the main parameters of data distribution structure are as follows: a median of $x_{\mathrm{M}}$, upper quantile $x_{\mathrm{U}}$, lower quantile $x_{\mathrm{L}}$, and quantile dispersion of $d_{\mathrm{F}}$. The dispersion is used to reflect the physical quantities of data dispersion. If the dispersion of data is small, the data will be considered to be normal. Otherwise, it is considered to be abnormal.

Suppose a vehicle receives speed information from ten neighbor nodes at the same time. Order the received data from small to large: $x_{1}, x_{2}, \ldots x_{9}, x_{10}$. Then, $x_{1}$ and $x_{10}$ correspond to the lower limit and the upper limit, respectively.

The median is defined as $x_{M}=\left(x_{5}+x_{6}\right) / 2$. Thus, the upper quantile $x_{8}$ is the median at interval $\left[x_{M}, x_{10}\right]$, and the lower quantile $x_{3}$ is the median at interval $\left[x_{1}, x_{M}\right]$. The quantile dispersion is calculated as $d_{\mathrm{F}}=x_{8}-x_{3}$. If the distance between the median and the data is longer than $k \times d_{\mathrm{F}}$, it belongs to the abnormal data. Here, $k$ is an arbitrary constant. We can limit the range of valid data by adjusting the value of $k$.

\section{Adaptive routing based on traffic flow phase}

Once a vehicle identifies its current traffic flow phase, it can copy the packets at different times and choose the corresponding number of next-hop nodes to forward them to obtain better performance. That is, for a much stable mobility environment, just one copy of the packets

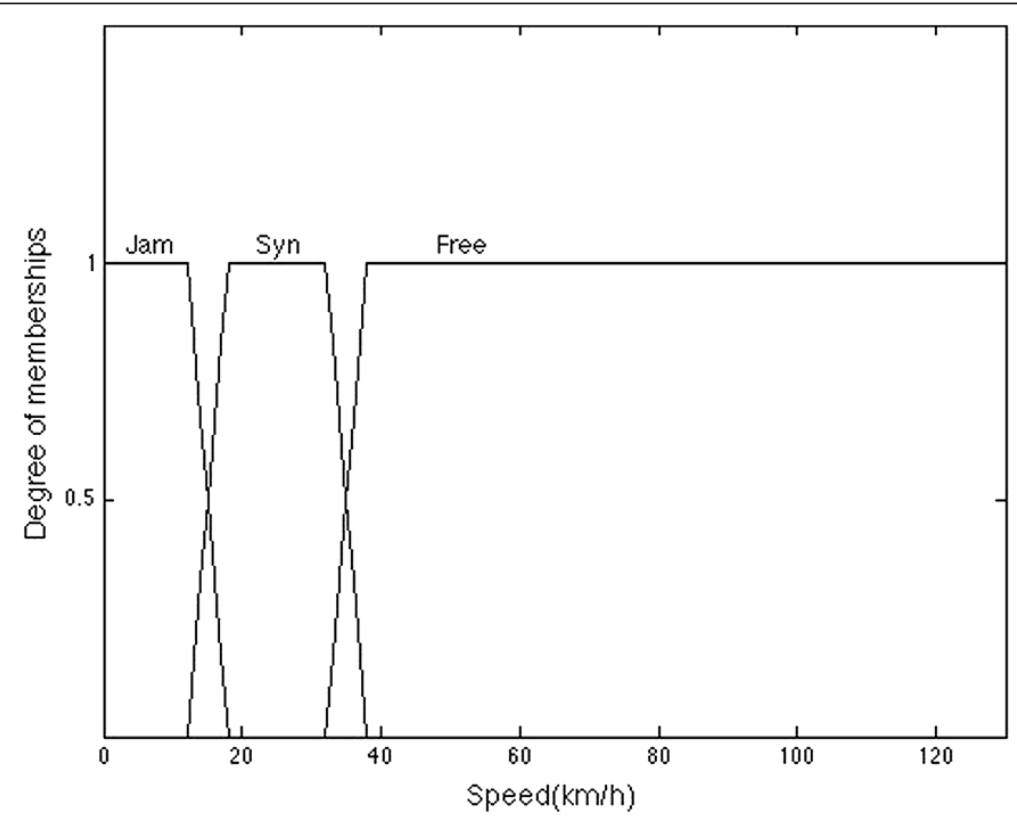

Figure 1 Membership function based on speed. The figure gives the membership functions of fuzzy sets based on vehicular speed. There are three different membership functions of fuzzy sets, which correspond to free flow, synchronized flow, and congested flow, respectively. 


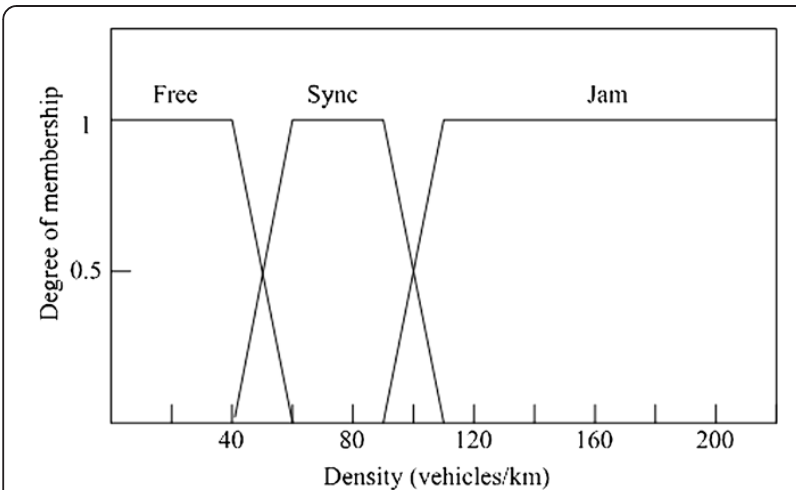

Figure 2 Membership function based on density. The figure gives the membership functions of fuzzy sets based on local density. There are three different membership functions of fuzzy sets, which correspond to free flow, synchronized flow, and congested flow, respectively.

is used to forward to only one next-hop node, which still can achieve good performance. Otherwise, the network connectivity is fragile. In that case, more than one copy of the packets will be used to forward to different next-hop nodes, which will help enhance transmission success ratio. The detailed procedure is given below.

\subsection{Data structures}

Each node maintains a Next-Hop Forwarding Link (NHFL) table (Table 1). It records the next-hop nodes which can participate in packet forwarding. It is maintained through periodic HELLO message. The maximum number of entries in the NHFL table is allowed to be $n$. That means, at most, $n$ nodes can be the candidates to forward packets. The entries for these nodes are sorted according to Section 4.3 , and the most suitable forwarding node will be put in the front. Once the first $m(1 \leq m \leq n)$ nodes are selected for forwarding, the packets will be copied $m$ times and be unicasted to these $m$ nodes separately. Maybe for a given node in a sparse environment, there is no HELLO message from neighbors during an interval, so the NHFL table can be null.

Each node also maintains a Packet Sent Out Information table, which records the packet ID and its left time, and also whether the packet is sent out or not (Table 2). Since a node still has a chance to receive the same copy of a packet which has been sent out already, it is unnecessary to send it again. If the lifetime expires, the packet will be discarded.

Table 1 Next-Hop Forwarding Link

\begin{tabular}{cccccc}
\hline ID & Position & Velocity & Moving direction & Timestamp & Lifetime \\
\hline$n_{1}$ & $\left(x_{1}, y_{1}\right)$ & $v_{1}$ & $\theta_{1}$ & $t_{1}$ & $\Delta t_{1}$ \\
$n_{2}$ & $\left(x_{2}, y_{2}\right)$ & $v_{2}$ & $\theta_{2}$ & $t_{2}$ & $\Delta t_{2}$ \\
\hline
\end{tabular}

Table 2 Packet Sent Out Information

\begin{tabular}{lll}
\hline Packet ID & Sent out & Lifetime \\
\hline$p_{1}$ & $1 / 0$ & $\Delta t_{1}$ \\
\hline
\end{tabular}

\subsection{Forwarding strategies}

Upon reception of a packet, if it is not the first time for a node to receive it, the node will just discard it. Otherwise, the node will adopt different forwarding strategies according to the traffic flow phase it resides in. If the node is in the free flow phase, it is said to be in sparsely connected neighborhood. Therefore, all the nodes (suppose there are $n$ nodes) in the NHFL table are used to forward packets, i.e., point-to-multipoint transmission will be conducted and all the neighbors may receive the packets possibly. If one copy is transmitted successfully, the reliability of transmission can be achieved. Maybe there is no neighbor for a given node during an interval, and then the node has to store the packet until a HELLO message is received from back vehicles or until the packet timer expires. The packet expiration time is a very important parameter in packet forwarding since it affects the reliability of packet reception. If a node is in the congested flow phase, the node is said to be in densely connected neighborhood. Therefore, only the first node in the NHFL table is used to forward packets, which means point-to-point mode is adopted in transmission. By this way, it can help reduce network congestion and avoid packet loss due to channel competition.

To compute the suitable number of forwarding nodes in the synchronous flow phase, the standard deviation of vehicle speed is calculated. If the standard deviation is above the predefined threshold and traffic density is low, which means network topology is highly dynamic, two copies of the packets will be transmitted to two different next-hop nodes separately. Otherwise, just one neighbor is chosen as a forwarding node.

\subsection{Computing the most suitable forwarding node}

Each time, the most suitable forwarding neighbors are selected based on the current traffic flow phase, which will be put into the NHFL table. The NHFL table is restricted to contain at most $n$ nodes, all of which are the candidate forwarding nodes. In the congested flow phase, since the topology is very stable, the node will select the neighboring node which is far from itself but still within the transmission range as the most suitable forwarding node. We can find $n$ candidates in such phase, but only the first one will participate in transmission. If a node is in the synchronous flow phase, the neighbors with the biggest link lifetime will be selected as the candidate forwarding nodes. To compute the link lifetime, mobility prediction is used and vehicles' velocity is assumed unaltered. The absolute distance between 


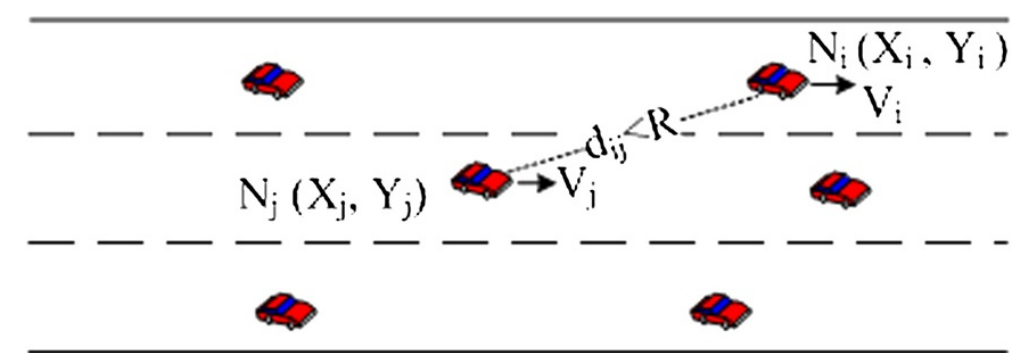

Figure 3 Link lifetime estimation based on mobility prediction. The figure illustrates how to compute the link lifetime based on mobility prediction with vehicles' velocity assumed unaltered.

two nodes $n_{i}$ and $n_{j}$ is denoted by $\left|d_{i j}\right|$, and their corresponding speeds are represented by $V_{i}$ and $V_{j}$, respectively. Their position coordinates are $\left(X_{i}, Y_{i}\right)$ and $\left(X_{i}, Y_{i}\right)$, respectively. Assume that $Y_{i}$ and $Y_{j}$ are very close, and thus, the gap between $Y_{i}$ and $Y_{j}$ can be neglected. The communication range of nodes in the wireless network is $R$. So as shown in Figure 3, the lifetime of link between node $n_{i}$ and $n_{j}$ is estimated as follows [14]:

$$
\text { Lifetime }_{i j}=\left\{\begin{array}{l}
\frac{R-\left|X_{i}-X_{j}\right|}{\left|V_{i}-V_{j}\right|}, V_{i}<V_{j} \\
\frac{R+\left|X_{i}-X_{j}\right|}{\left|V_{i}-V_{j}\right|}, V_{i}>V_{j}
\end{array}\right.
$$

If a node is in the free flow phase, the neighbors around a given node will be rare and the neighborship may be changed rapidly. Therefore, all the vehicles that move into the transmission range of the node will be selected as the forwarding nodes.

\section{Simulation and discussion}

The simulation tools used in this paper include Simulation of Urban Mobility (SUMO) [14], Mobility Model Generator for VANET (MOVE) [15], Network Simulator 2 (NS2) [16], and Matlab, where SUMO and MOVE are used to model the moving behavior of vehicles and produce traffic information such as velocity and location, NS2 is used to simulate communication procedure and analyze network performance, and Matlab is used to calculate and process simulation data locally. The relationship of these tools is illustrated in Figure 4.

A highway scenario is set with a length of 5,000 $\mathrm{m}$ and two lanes. The number of vehicles varies from 50 to 90 , and their moving speed changes from 10 to $100 \mathrm{~km} / \mathrm{h}$. The radio frequency uses $5.9 \mathrm{GHz}$, and the communication range is $250 \mathrm{~m}$. A HELLO message is broadcasted periodically at an interval of $1 \mathrm{~s}$, and the packet size is 512 bytes. The whole simulation runs for $300 \mathrm{~s}$.

\subsection{Simulation of traffic state estimation}

Assume that each vehicle is equipped with GPS and VANET communication equipment (i.e., 802.11a), which are capable of processing basic data. In the simulation scenario, a vehicle broadcasts the HELLO message to its neighbors among its communication range. Since the HELLO message includes the information about its own speed and location information, a vehicle can calculate the number of vehicles and their speeds after exchanging HELLO message with its neighbors. Then, a designated vehicle will remove the abnormal data by using the distribution diagram method. Here, $k$ is set to 2 . Then, it

NS2

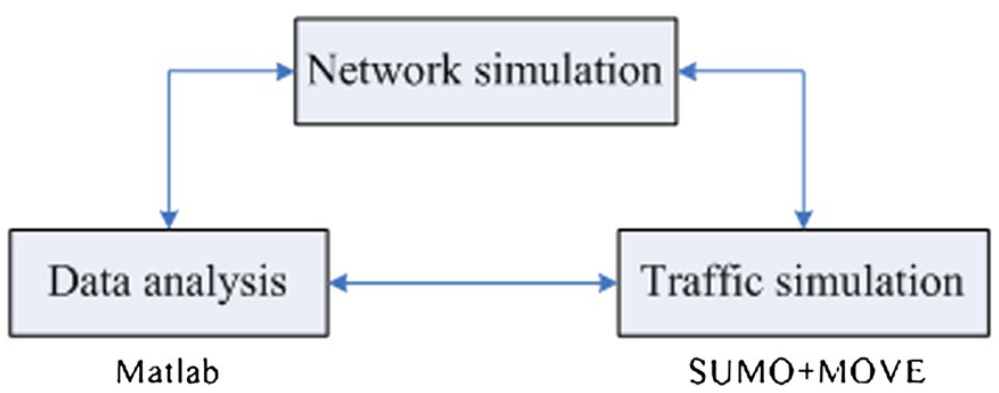

Figure 4 Simulation architecture. The figure presents the simulation tools used in this paper and the relationship among these tools. 


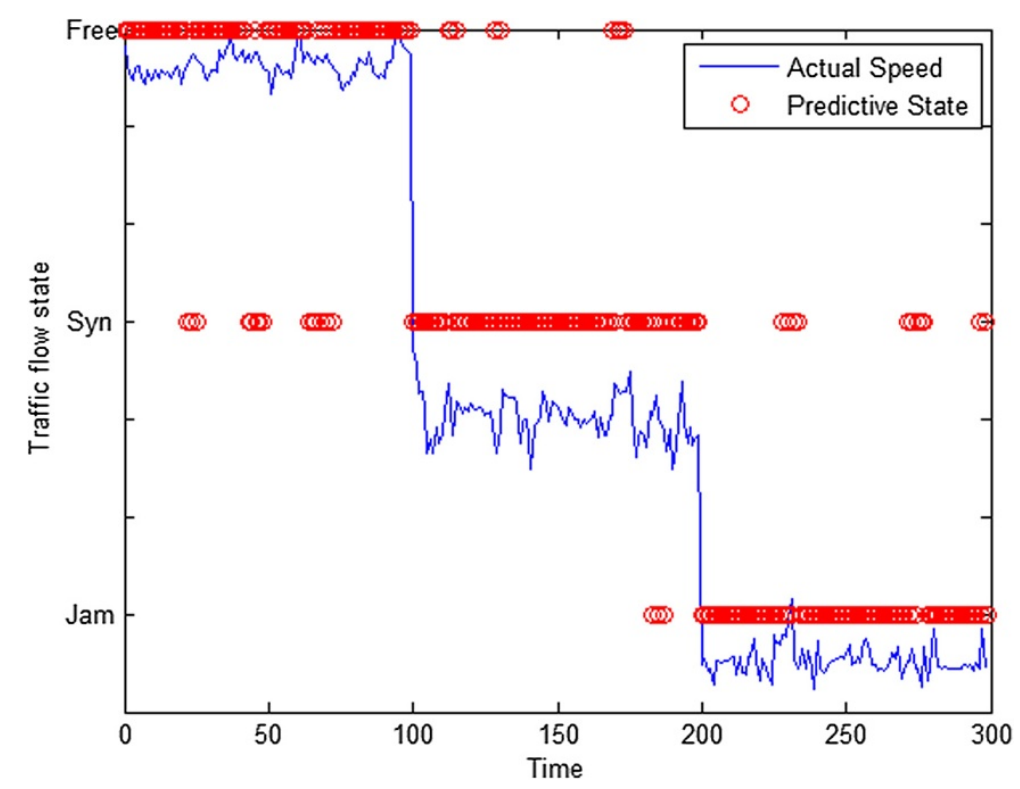

Figure 5 The predictive traffic state vs. actual speed. The figure illustrates the results of predictive traffic states compared with actual vehicle speed. The state prediction is based on the proposed self-organizing method, and only the factor of vehicle speed is considered.

will process the valid data to get the average speed and density of current local traffic among its communication range. The threshold for speed standard deviation is defined 35 . We record the change of speed and density and the corresponding phase output at the same time under three traffic flow scenarios.

Firstly, the results of predictive traffic states are compared with actual traffic speed and actual traffic density, separately. In this paper, we design three traffic flow scenarios according to Kerner's three-phase traffic model. There is a large difference among these three phases in terms of traffic speed and density which is in line with our perception of traffic phases. By using the membership function given in Figures 1 and 2 and the prediction method of fuzzy logic in Equation 1 with the weighting factor $\alpha(=0.7)$, the output curve of the traffic phases can

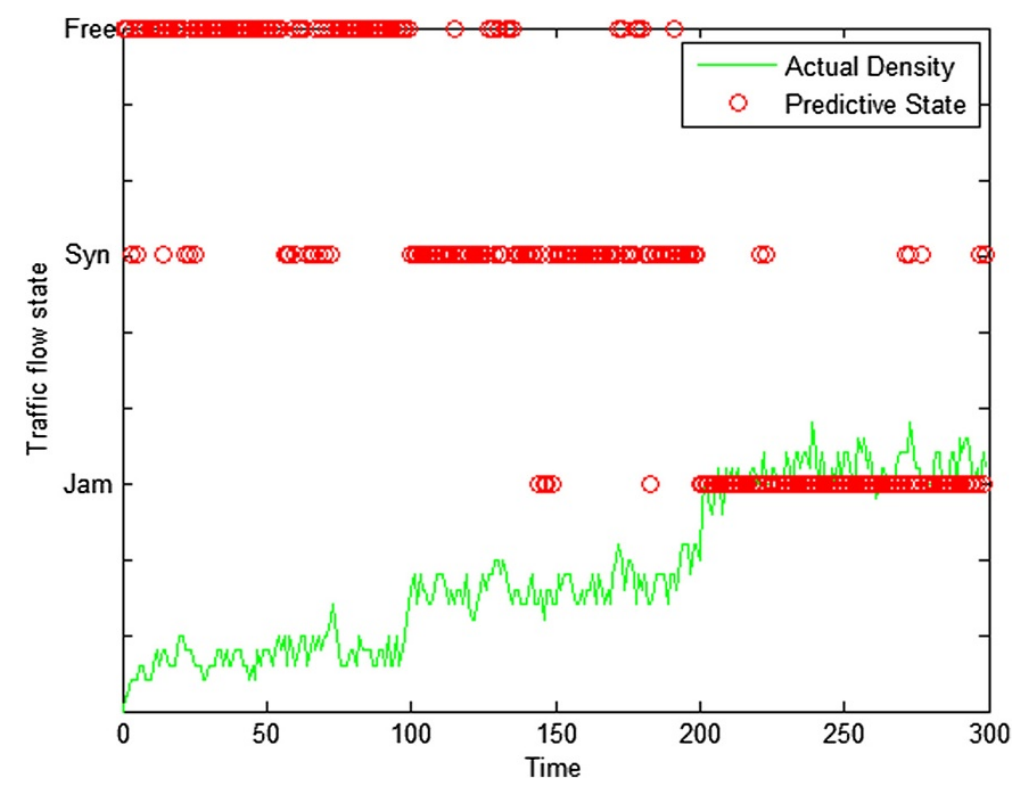

Figure 6 The predictive traffic state vs. actual density. The figure illustrates the results of predictive traffic states compared with actual traffic density. The state prediction is based on the proposed self-organizing method, and only the factor of local density is considered. 


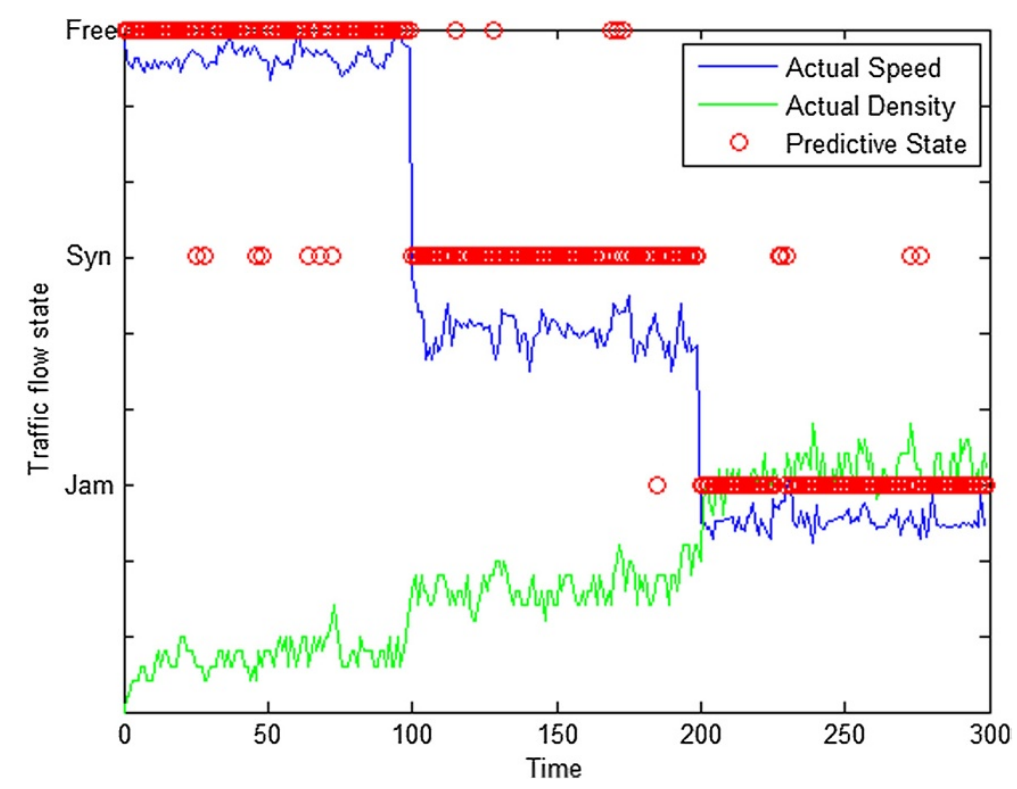

Figure 7 The predictive traffic state vs. actual speed and actual density. The figure illustrates the results of predictive traffic states compared with actual vehicle speed and traffic density. The state prediction is based on the proposed self-organizing method, and the factors of vehicle speed and local density are considered together.

be plotted as shown in Figures 5 and 6. It can be seen that there is a higher recognition rate in the current traffic flow phase, but there are some wrong judgments due to the large variation of speed or density. This also shows that it is not comprehensive when adopting a single parameter of traffic characteristics to judge the phase of traffic flow.

After considering the speed and density comprehensively, the impact of amplitude change of a single factor on misjudgment of the traffic flow phase is weakened. It can be seen from Figure 7 that the recognition rate of traffic flow in three traffic phases has been greatly improved. But at the same time, there are still errors at some points. The possible reason is that these points are misjudged ones, both in the membership function of speed and the one of density. We believe that if more traffic factors are considered, such as speed deviation, the

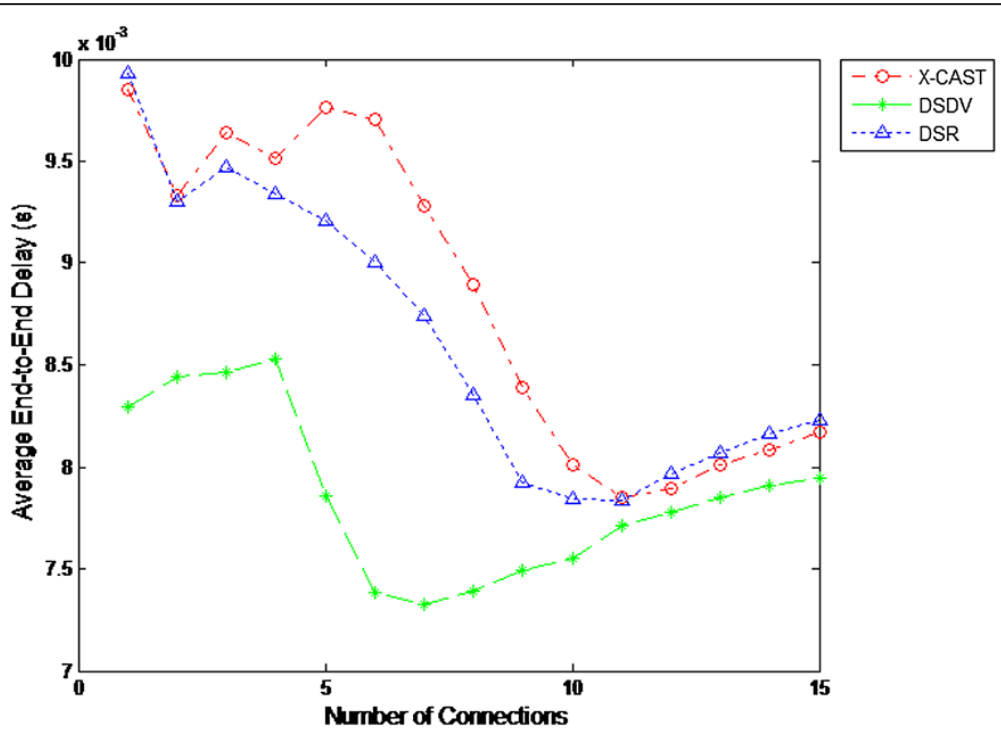

Figure 8 Average end-to-end delay in dense traffic flow. The figure shows the average end-to-end delay with respect to the number of CBR connections in density scenario with 50 vehicles $/ \mathrm{km}$, where the phase of traffic flow may transfer from congested flow to synchronized flow. 


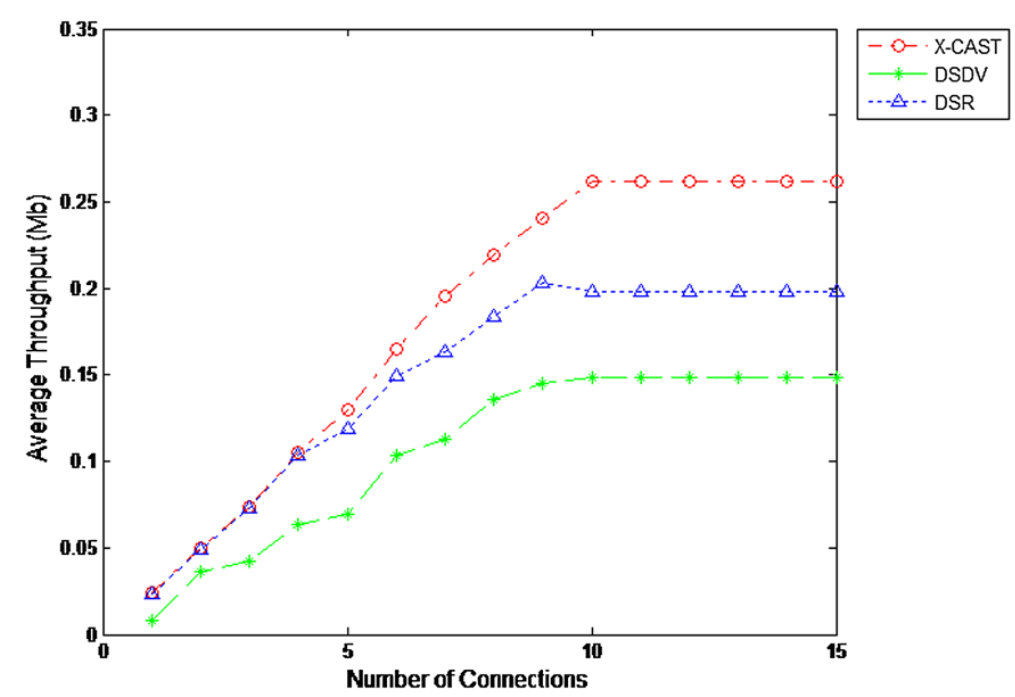

Figure 9 Average throughput in dense traffic flow. The figure shows the average throughput with respect to the number of CBR connections in density scenario with 50 vehicles $/ \mathrm{km}$, where the phase of traffic flow may transfer from congested flow to synchronized flow.

error rate can be further reduced. In other words, the method proposed in this paper can identify the phase of traffic flow very well.

\subsection{Evaluation of forwarding performance}

To analyze the performance of the proposed routing algorithm, two different scenarios are considered. One is density scenario with 50 vehicles $/ \mathrm{km}$, where the phase of traffic flow may transfer from congested flow to synchronized flow; the other is sparse scenario with 5 vehicles/ $\mathrm{km}$, where the phase of traffic flow may transfer from free flow to synchronized flow. Traffic type was constant bit rate (CBR) with 512 bytes of packet size and $512 \mathrm{kbps}$ of maximum CBR. CBR traffic sources are increased from 1 to 15.

The communication performance metrics studied here are as follows:

- Packet loss rate - the ratio of the data packets each routing protocol loses to those originated by an application

- End-to-end delay of a packet - the average difference between the time a data packet is originated by an application and the time this packet is received at its destination

- Throughput - the average amount of messages, which are successfully delivered and received

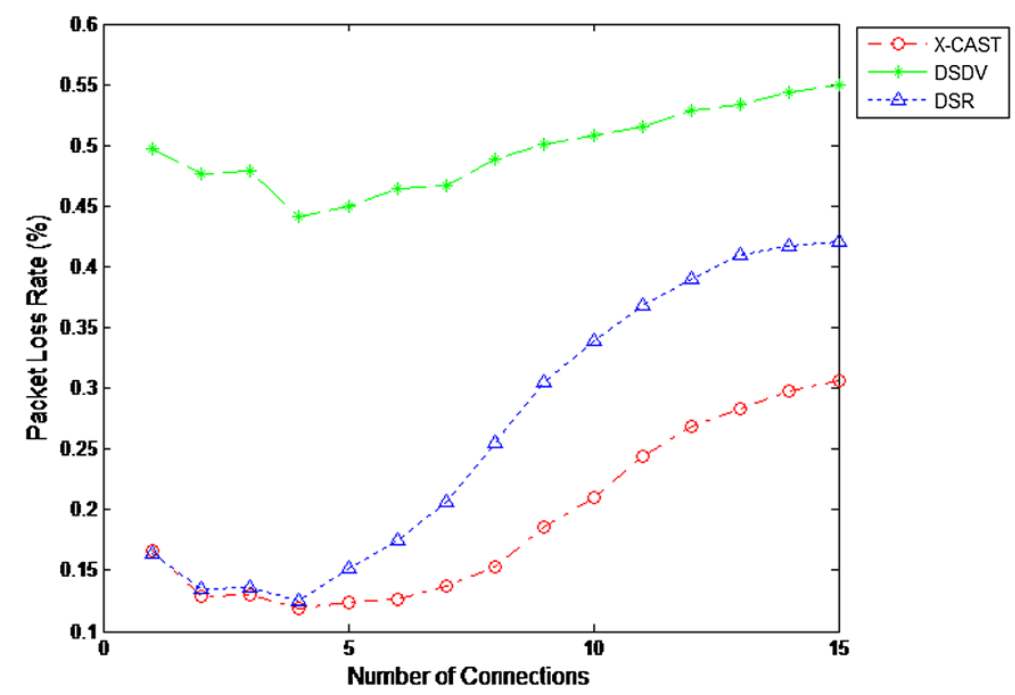

Figure 10 Packet loss rate in dense traffic flow. The figure shows the packet loss rate with respect to the number of CBR connections in density scenario with 50 vehicles/km, where the phase of traffic flow may transfer from congested flow to synchronized flow. 


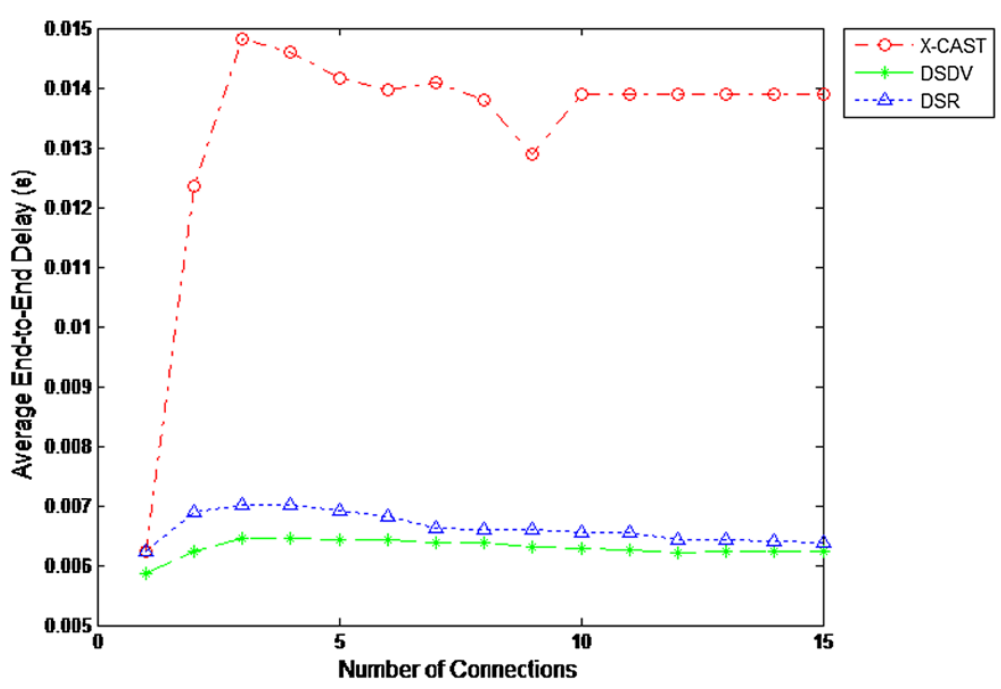

Figure 11 Average end-to-end delay in sparse traffic flow. The figure shows the average end-to-end delay with respect to the number of CBR connections in sparse scenario with 5 vehicles $/ \mathrm{km}$, where the phase of traffic flow may transfer from free flow to synchronized flow.

The different numbers of CBR connections are used in simulation. The performance of the proposed algorithm (here called X-CAST) will be compared with those of destination-sequenced distance vector routing (DSDV) [17] and dynamic source routing (DSR) [18]. DSDV is a typical proactive routing protocol, where each node has to maintain one or more route tables to maintain the latest information of the routes to any valid node in the network. DSR is one of the more generally accepted reactive routing protocols, where routes are established only when they are needed to send packets to a destination, and these routes expire after some time. First, the performance in the dense traffic flow scenario is analyzed. Figure 8 shows the average end-to-end delay with respect to the number of CBR connections. The figure shows that the delay of XCAST is slightly higher than those of DSDV and DSR, although all of them are low. The reason is maybe that XCAST needs to accomplish the process of state prediction and link lifetime prediction, and this calculation will cost some processing time.

As shown in Figures 9 and 10, in dense traffic flow scenario, X-CAST performs better compared with DSDV and DSR in terms of throughput and packet loss rate. A vehicle in such scenario may be residing in the

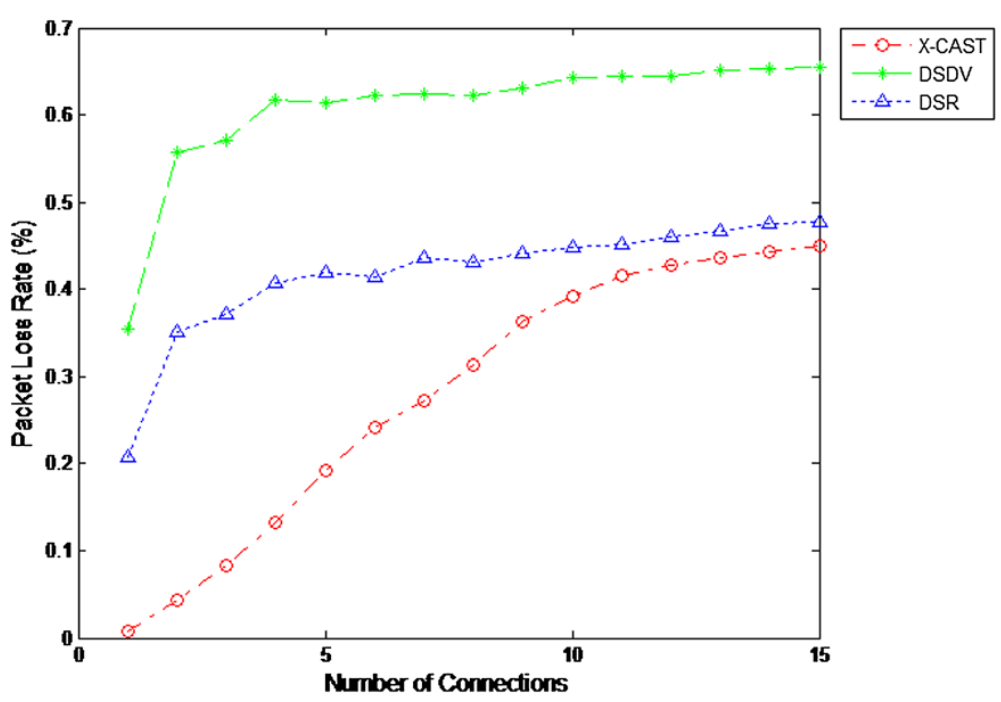

Figure 12 Packet loss rate in sparse traffic flow. The figure shows the packet loss rate with respect to the number of CBR connections in sparse scenario with 5 vehicles $/ \mathrm{km}$, where the phase of traffic flow may transfer from free flow to synchronized flow. 
congested flow phase or synchronized phase. If a vehicle is in the synchronized phase, more than one copy of the packets is transmitted to the different next-hop nodes separately, which not only increases the success ratio of packet delivery, but also reduces network overhead. If a vehicle is in the congested flow phase, the next-hop forwarding node selection in X-CAST is similar to greedy mode, so that the number of hops a packet needed to be forwarded before it arrives at the destination is fewer than others. That means fewer relays will participate in packet forwarding, which also contributes to throughput.

Figures 11, 12, and 13 gives the simulation results under the sparse traffic flow scenario. As shown in Figure 11, the average end-to-end delay in X-CAST is bigger than those of DSDV and DSR, but still remains in an acceptable level. As far as packet loss rate and throughput are concerned, X-CAST shows much better performance compared with DSDV and DSR, as shown in Figures 12 and 13. In the sparse traffic flow scenario, a vehicle may be residing in the free flow phase or synchronized flow phase. For the former case, more than one copy of the packets is transmitted in X-CAST if more than one neighbor is available, which helps increase the successful probability of packet delivery. For the latter case, X-CAST easily establishes a more stable path among moving vehicles; thus, packets are not easy to be discarded during the period of transmission.

\section{Conclusion}

The prediction and identification of traffic phase are basic and important subjects in the field of intelligent transport systems. Unlike the traditional way which relies on the ground coils or other infrastructures to obtain traffic information, vehicles can obtain traffic information through the platform of vehicular ad hoc networks, which does not rely on any infrastructures, and thus, it makes traffic information collection and distribution become simple and timely. This is helpful to carry out the related applications based on real-time traffic status, such as dynamic path planning. After the traffic data are processed and analyzed, a new method for identifying traffic flow phases is proposed based on the membership function of fuzzy logic. The method is simple and easy to implement, so it is extremely suitable for vehicle communication networks. The simulation results show that the method gains satisfactory accuracy in identifying traffic flow phase. When more factors are considered during traffic flow prediction, we can further improve the accuracy and practicality of the algorithm.

Once real-time traffic conditions are available, an adaptive routing algorithm X-CAST is proposed for vehicular communication on highways. According to different traffic flow phases, X-CAST will transmit different copies of the packets to the next-hop nodes separately and the next forwarding nodes will be chosen based on traffic flow. The proposed X-CAST scheme is compared with traditional routing protocols DSR and DSDV. The results show that X-CAST performs much better both in low and high vehicle density. Unfortunately, a comprehensive scenario which includes all the three flow phases simultaneously is not simulated. In one simulation, a scenario where a vehicle experiences the transition not only from the congested flow to the synchronized flow, but also from the synchronized flow to the free flow or vice versa can testify the performance more factually.

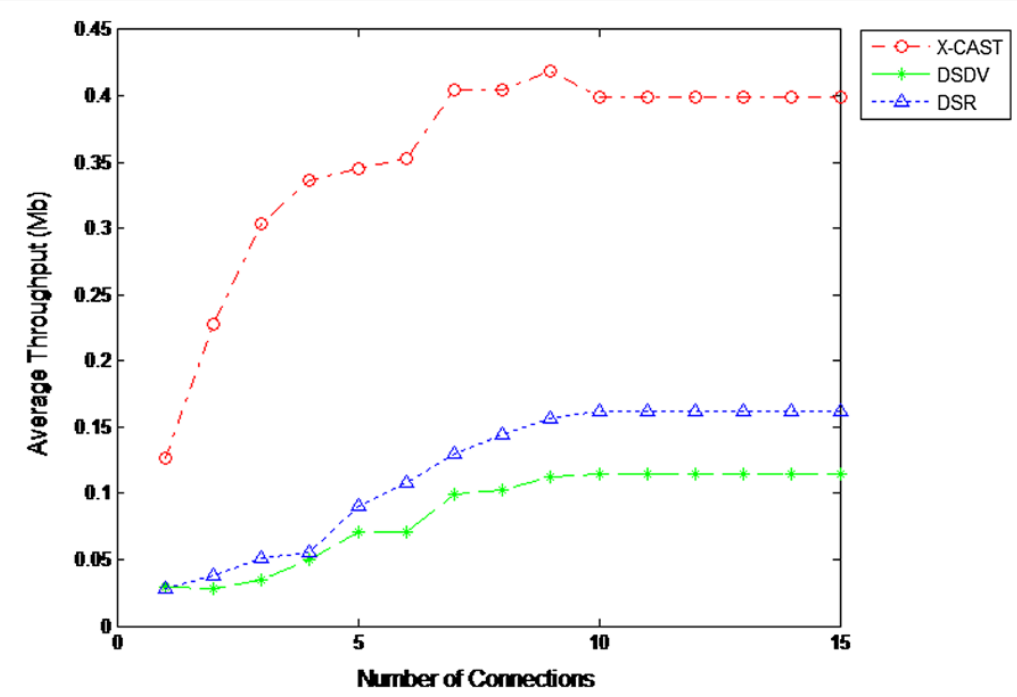

Figure 13 Average throughput in sparse traffic flow. The figure shows the average throughput with respect to the number of CBR connections in sparse scenario with 5 vehicles $/ \mathrm{km}$, where the phase of traffic flow may transfer from free flow to synchronized flow. 


\section{Competing interests}

The authors declare that they have no competing interests.

\section{Acknowledgements}

This work was supported by the National Natural Science Foundation of China under the Grant No. 61103179.

\section{Author details}

'Broadband Wireless Communication and Multimedia Laboratory, College of Electronics and Information Engineering, Tongji University, Shanghai 201804, China. ${ }^{2}$ School of Electronics and Telecommunications, Hanoi University of Science and Technology, Hanoi, Vietnam.

Received: 18 November 2013 Accepted: 18 February 2014 Published: 12 March 2014

\section{References}

1. J Chennikara, W Chen, S Cai, Survey of routing protocols for inter-vehicle communications, paper presented at the 3rd Annual International Conference on Mobile and Ubiquitous Systems-Workshops, 2006, pp. 1-5

2. W Chen, S Cai, Ad hoc peer-to-peer network architecture for vehicle safety communications. IEEE Commun. Mag. 43(4), 100-107 (2005)

3. F-Y Wang, C Herget, D Zeng, Developing and improving transportation systems: the structure and operation of IEEE intelligent transportation systems society. IEEE Trans. Intell. Transport. Syst 6(3), 261-264 (2005)

4. L Figueiredo, I Jesus, JAT Machado, JR Ferreira, JLM de Carvalho, Towards the development of intelligent transportation systems, paper present, in Proc. IEEE Intelligent Transportation Systems, 2001, pp. 1206-1211

5. B Ducourthial, Y Khaled, M Shawky, Conditional transmissions: a communication strategy for highly dynamic vehicular ad hoc networks. IEEE Trans. Vehicular Commun. 56(6), 3348-3357 (2007)

6. G Resta, P Santi, J Simon, Analysis of multi-hop emergency message propagation in vehicular ad hoc networks (Proceedings of the 8th ACM International Symposium on Mobile Ad Hoc Networking and Computing (MobiHoc '07), New York, USA, 2007), pp. 140-149

7. M Jerbi, S Senouci, T Rasheed, Y Ghamri-Doudane, An infrastructure-free traffic information system for vehicular networks (Proceedings of 2007 IEEE 66th Vehicular Technology Confence (VTC2007 Fall), Baltimore, MD, USA, 2007), pp. 2086-2090

8. S Panichpapiboon, W Pattara-atikom, Evaluation of a neighbor-based vehicle density estimation scheme, paper presented in the 8th International Conference on ITS Telecommunications (ITST 2008), 2008, pp. 294-298

9. $Y X u, Y$ Wu, J Xu, D Ni, G Wu, L Sun, A queue-length-based detection scheme for urban traffic congestion by VANETS, paper presented in the 7th International Conference on Networking, Architecture and Storage (NAS), 2012, pp. 252-259

10. BS Kerner, Introduction to Modern Traffic Flow Theory and Control, The Long Road to Three-Phase Traffic Theory (Springer-Verlag Berlin, New York, 2009)

11. MM Artimy, W Robertson, WJ Phillips, Assignment of dynamic transmission range based on estimation of vehicle density (Proceedings of the 2 nd ACM International Workshop on Vehicular Ad Hoc Networks (VANET '05), New York, USA, 2005), pp. 40-48

12. O Tonguz, N Wisitpongphan, F Bai, P Mudalige, V Sadekar, Broadcasting in VANET, paper presented in Proceedings of 2007 Mobile Networking for Vehicular Environments workshop, 2007, pp. 7-12

13. LA Zadeh, Fuzzy Sets. Inf. Control. 8(3), 338-353 (1965)

14. SUMO, Simulation of Urban Mobility, 2006. http://sumo.sourceforge.net. Accessed 13 Aug 2006

15. MOVE, Mobility Model Generator for VANET, 2003. http://lens1.csie.ncku.edu.tw/ Joomla_Version/index.php/resources. Accessed 3 July 2003

16. The Network Simulator: NS-2, 2005. http://www.isi.edu/nsnam/ns. Accessed 12 Oct 2005
17. C Perkins, P Bhagwat, Highly dynamic destination-sequenced distancevector routing (DSDV) for mobile computers. ACM SIGCOMM Computer Communication Review. 24(4), 234-244 (1994)

18. DB Johnson, Y-C Hu, D Maltz, The dynamic source routing protocol (DSR) for mobile ad hoc networks for IPV4 (IETF Network Working Group, RFC4728, 2007). https://www.ietf.org/rfc/rfc4728.txt. Accessed 28 Feb 2007

doi:10.1186/1687-1499-2014-38

Cite this article as: Wang et al:: A traffic flow phase adaptive routing for vehicular communication on highways. EURASIP Journal on Wireless

Communications and Networking 2014 2014:38.

\section{Submit your manuscript to a SpringerOpen ${ }^{\odot}$ journal and benefit from:}

- Convenient online submission

- Rigorous peer review

- Immediate publication on acceptance

- Open access: articles freely available online

- High visibility within the field

- Retaining the copyright to your article

Submit your next manuscript at $>$ springeropen.com 